\title{
Aprendizaje Basado en Proyectos en asignaturas de Ciclo de Vida del Producto en estudios de Máster de Diseño y Fabricación
}

\author{
Norberto Feito $^{\text {a }}$, Ricardo Belda ${ }^{\mathrm{a}}$, Raquel Megías ${ }^{\mathrm{a}}$ y Carlos Vila \\ a Departamento de Ingeniería Mecánica y de Materiales, Universitat Politècnica de València, Camino de Vera s/n, \\ 46022, Valencia, España, norfeisa@upv.es, ribelgon@upv.es, ramedia@upv.es y carvipas@upv.es
}

\section{\$EWWDWW}

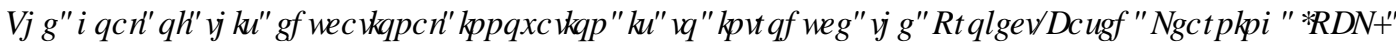

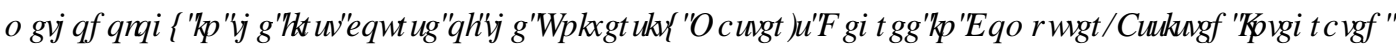

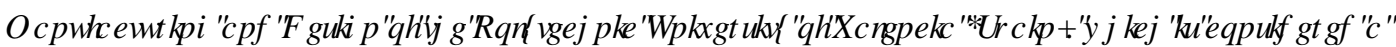

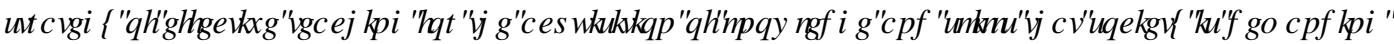

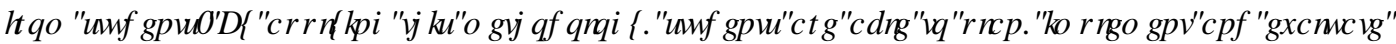

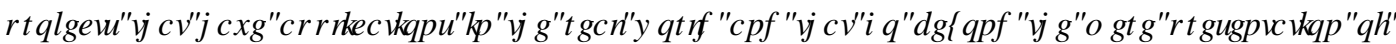

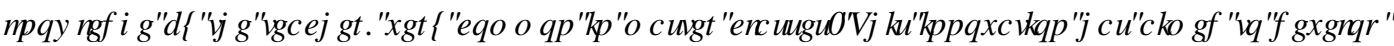

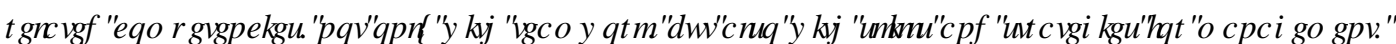

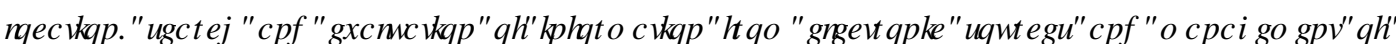

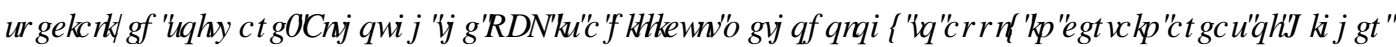

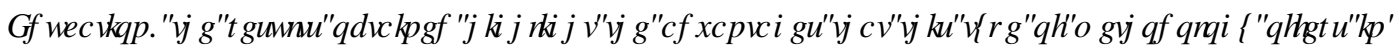

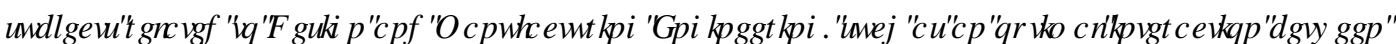

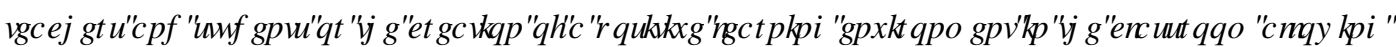

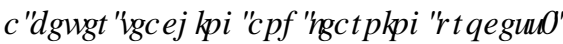

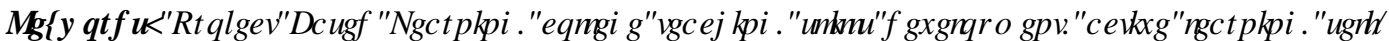
ODQTQTI [

\section{HXP HQ}

( Q RENAMWR GH HMD IQQRYDFIYQ GRFHQUI KD UGR IQNRGXFIU HQ HD SUP HU FXURD GHD 0 i UAU

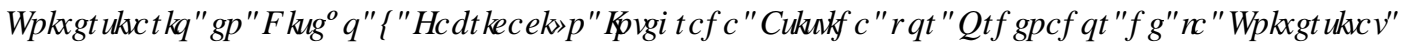

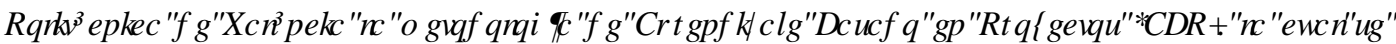
FRQMGHD XQD HWDUID GH HQMKDQ]D HIFD] SDW QD DGTXIMFIYQ GH RRV FRQRFP IHQURVI I

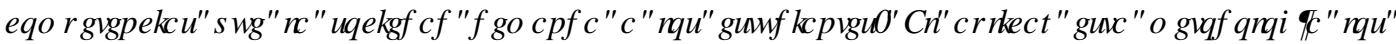

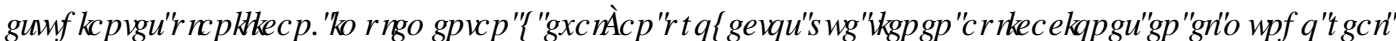

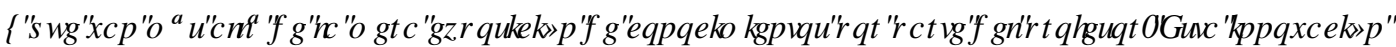

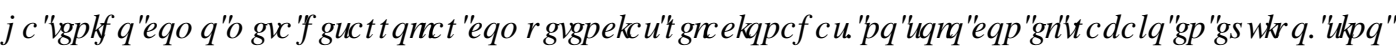

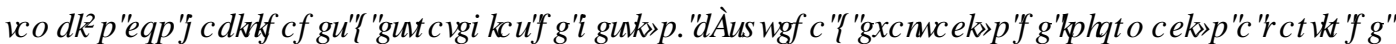

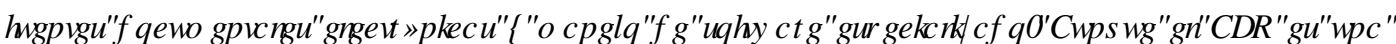

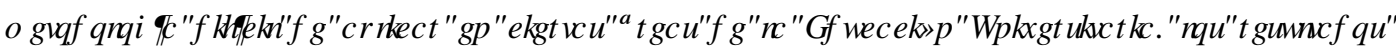

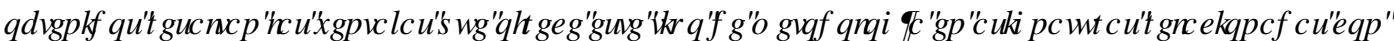

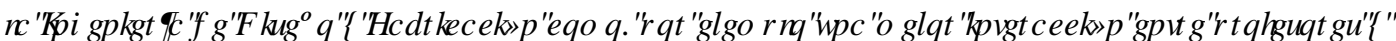

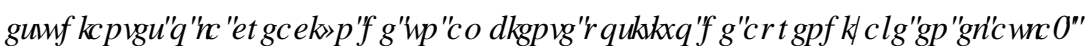

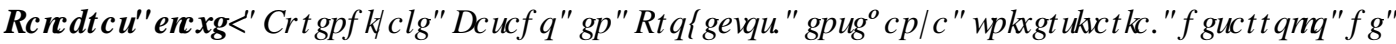
KDEIDCDOHMDSUHQG] DVAIDFWYRTDXURDSUHQG]DNAII 


\section{Introducción}

Uno de los principales aspectos novedosos del Espacio Europeo de Educación Superior (EEES), que se puso en marcha en 2010, es el establecimiento de un sistema educativo basado en el aprendizaje en vez de en la enseñanza. Es decir, el protagonista del proceso educativo debe ser el alumno, quien tendrá un aprendizaje activo, y no el profesor, que se convierte en un el facilitador del aprendizaje. Esto supone pasar de un sistema basado en la simple transmisión de conocimientos, a otro en el que el alumno no sólo aprenda los conocimientos propios de la asignatura, sino que, además, adquiera una serie de competencias adicionales que le serán de utilidad en el futuro ejercicio de su profesión (García, 2018). Por lo tanto, se puede decir que el nuevo perfil académico está orientado a la adquisición del conocimiento y a la práctica profesional.

Ante este nuevo escenario del EEES, la metodología de Aprendizaje Basado en Proyectos (ABP) es considerada una metodología muy adecuada a implementar en el ámbito de la Educación Superior, ya que permite trabajar las competencias profesionales determinantes en el perfil del estudiante universitario, y es por eso que cada vez más docentes la aplican en sus asignaturas, ya sean de Grado, (Estepa y Estepa, 2011; Machado y Berges, 2013) o de Máster (Garrigós y Valero-García, 2012; Gonçalves, 2014).

\subsection{Aprendizaje Basado en Proyectos}

El tipo de aprendizaje a través de la implementación de proyectos se denomina "Aprendizaje Basado en Proyectos" (ABP). Con la aplicación de esta estrategia, los estudiantes definen el propósito de la creación de un producto final, identifican su mercado, investigan la temática, crean un plan para la gestión del proyecto y diseñan y elaboran un producto. Ellos comienzan el proyecto solucionando problemas, hasta llegar a su producto.

El proceso completo es auténtico, referido a la producción en forma real, utilizando las propias ideas de los estudiantes y completando las tareas en la práctica (Mettas, 2007). Dentro de este marco, los alumnos persiguen soluciones a problemas no triviales, generando y refinando preguntas, debatiendo ideas, realizando predicciones, diseñando planes y/o experimentos, recolectando y analizando datos, estableciendo conclusiones, comunicando sus ideas y resultados a otros, realizando nuevas preguntas y creando o mejorando productos y procesos (Blumenfeld, 1991).

En los últimos años se han publicado estudios donde se ha demostrado la eficacia del ABP para trabajar conjuntamente competencias específicas y competencias transversales de la asignatura o asignaturas a las que aplique, así como para preparar al estudiante para su futuro desarrollo profesional (Cuiñas, 2016; Toleado, 2018). De estos resultados se desprende que la metodología docente aplicada tiene efectos positivos sobre el rendimiento académico y la motivación del alumnado (Zafra, 2020).

Es importante que el contenido del proyecto sea significativo para los alumnos y esté directamente conectado con su realidad. El ABP "engancha" al alumno porque éste siente que lo que aprende le es cercano y es importante para él. Partir de sus intereses y necesidades es esencial para conseguir el éxito del proyecto. El constructivismo se basa en el aprendizaje apoyado en conocimientos o ideas previas. En el $\mathrm{ABP}$ es fundamental partir de lo que ya conocen los alumnos, que ellos puedan "agarrar" el contenido y no sea todo absolutamente desconocido y nuevo (Pereira,2015). 


\subsection{La asignatura "Ciclo de Vida del Producto"}

La asignatura Ciclo de Vida del Producto tiene carácter optativo y se imparte en el segundo semestre del primer año del Máster Universitario en Diseño y Fabricación Integrada Asistidos por Computador (MUDFIAC) de la Universitat Poltècnica de València.

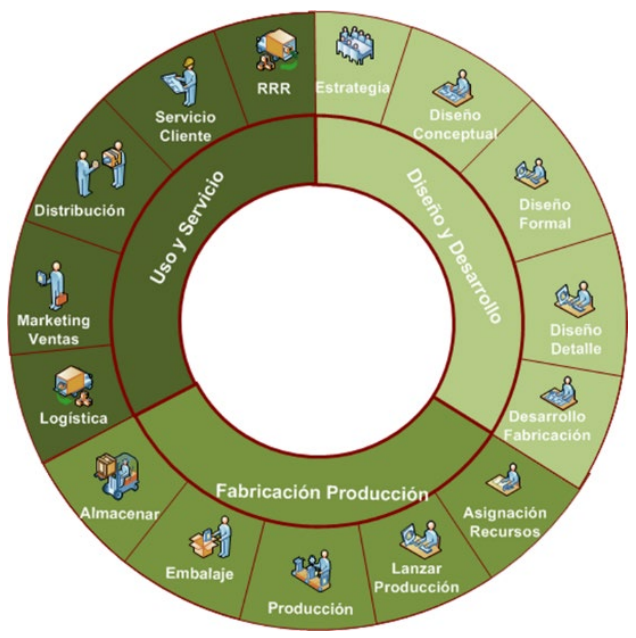

Según la descripción de la materia, el objetivo principal de la asignatura es que el alumno adquiera las destrezas para combinar los conocimientos de ingeniería con aplicaciones integradas para la gestión del ciclo de vida del producto. Como parte de la Ingeniería Concurrente, la integración de estas aplicaciones permite no sólo diseñar productos, analizar comportamientos y simular procesos de conformado sino, además, gestionar todo el proceso durante el ciclo de vida (Figura 1).

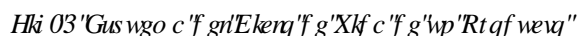

El objetivo mismo de la asignatura favorece la implementación de la metodología de ABP como la más idónea, tanto por su carácter multidisciplinar, que implica la integración y colaboración de diferentes áreas de ingeniería durante las etapas de diseño y desarrollo de un producto, como por la necesidad del alumno de aprender a utilizar las nuevas tecnologías de la información y comunicación para ser más competitivo (Gómez-Pablos, 2016). El desarrollo de las tecnologías web, junto con las modernas aplicaciones CAx, permite gestionar adecuadamente estas interacciones entre equipos de trabajo que pueden encontrarse geográficamente dispersos. Además, la capacidad de gestionar proyectos distribuidos, flujos de trabajo, tareas y carpetas compartidas será un valor añadido para los futuros ingenieros y están en línea con las competencias que se espera evaluar en la asignatura.

\section{Objetivos}

El objetivo general del trabajo es el de diseñar actividades enfocadas al Aprendizaje Basado en Proyectos y, definir técnicas de evaluación apropiadas centradas en el aprendizaje basado en proyectos en la asignatura de "Ciclo de Vida del Producto", potenciando el uso de los conceptos aprendidos en asignaturas durante el Grado como "Sistemas de Producción y Fabricación", "Ciencia de los Materiales" y "Expresión Gráfica".

Los objetivos específicos son:

- Diseñar actividades específicas en la asignatura de "Ciclo de Vida del Producto" de forma que se integre de forma transversal con los objetivos de aprendizaje de diversas asignaturas del Grado de Ingeniería Mecánica.

- Proponer experiencias prácticas más próximas a las aplicaciones reales que tendrán que emplear los estudiantes cuando trabajen como ingenieros.

- Observar y cuantificar de forma objetiva los resultados obtenidos de la implantación de esta metodología. 
Todos los objetivos planteados estuvieron dirigidos al desarrollo y evaluación de las competencias de aprendizaje en los estudiantes que quedan establecidas en el programa de la asignatura presentadas a continuación:

- Poder comunicarse y entender los procesos implicados en el desarrollo de un producto.

- Conocer las herramientas y técnicas para la integración de la fabricación en el desarrollo sostenible.

- Poseer y comprender conocimientos que aporten una base u oportunidad de ser originales en el desarrollo y/o aplicación de ideas, a menudo en un contexto de investigación.

- Gestionar el conocimiento para el diseño y la fabricación de productos.

- Diseñar, dirigir y evaluar una idea de manera eficaz hasta concretarla en un proyecto.

- Trabajar y liderar equipos de forma efectiva para la consecución de objetivos comunes, contribuyendo al desarrollo personal y profesional de los mismos.

\section{Desarrollo de la Innovación}

Durante los últimos cursos, se viene observando que los alumnos presentan problemas a la hora de aplicar los conceptos teóricos vistos en diversas asignaturas del Grado a problemas reales presentados en la asignatura de Máster. Por ejemplo, los alumnos conocen las propiedades de los diferentes materiales, pero no saben que material seleccionar para un determinado producto. Igualmente, conocen los procesos de fabricación más común en la industria, pero no saben seleccionar el proceso adecuado en función de criterios económicos. Este problema implica perder oportunidades de sinergia entre las distintas asignaturas para trabajar de forma más efectiva algunas competencias.

En este contexto, se busca que el alumno aplique los principios teóricos y prácticos impartidos en cada una de las asignaturas, organizándolos de manera global en una única propuesta, demostrando, tanto en el planteamiento, como en el desarrollo, una asimilación de conocimientos que indiquen un nivel a la altura de las exigencias profesionales. La finalidad del trabajo es abordar de forma integradora las distintas áreas de conocimiento en el proceso de diseño y desarrollo de un producto durante su ciclo de vida utilizando las tecnologías de la información y comunicación a través del aprendizaje basado en proyectos.

\subsection{Creación de grupos}

El primer paso consistió en formas los grupos de trabajo. Para ello se pasó un cuestionario a los alumnos antes de comenzar la asignatura con el objetivo de observar la formación previa que tienen los estudiantes cuando llegan al MUDFIAC. En este cuestionario se recoge, no solo la titulación y universidad de origen, sino que se pregunta sobre la formación que puede tener el alumno sobre el manejo de aplicaciones relacionadas con el diseño conceptual, el diseño asistido por ordenador, la ingeniería asistida por ordenador y la fabricación asistida por ordenador.

Con la información recabada, se formaron grupos de trabajo de 3 alumnos. De este modo se consiguieron grupos multidisciplinares en los que los integrantes no habían tenido relaciones entre sí, recreando la situación del egresado cuando entra a formar parte de una empresa por primera vez en su carrera laboral. A partir de aquí, se proporcionará a cada grupo un conjunto de especificaciones y requisitos preliminares de un producto a fabricar del que se deberá diseñar, planificar y simular el proceso de fabricación. 


\subsection{Planteamiento del problema}

Como se mencionó anteriormente, el papel del profesor en el Aprendizaje Basado en Proyectos es presentar el proyecto y sugerir varias tareas destinadas a guiar la investigación de los estudiantes. Se espera que los estudiantes asuman progresivamente el liderazgo del trabajo. De este modo, el proyecto a desarrollar se planteó en la primera clase, y duró todo el semestre dedicando una hora y media de las 3 horas semanales que tiene la asignatura, al desarrollo del proyecto.

En la actividad propuesta, cada grupo de trabajo tendrá que diseñar una mesa humidificadora, purificadora y deshumidificadora de aire con filtro HEPA. Los requisitos del proyecto planteado son los siguientes:

- Envolvente máxima de 50 x 50 x $50 \mathrm{~cm}$.

- Debe incluir como mínimo dos salidas de aire.

- Debe tener un depósito de $500 \mathrm{ml}$ que tenga fácil acceso de llenado.

- Debe incluir un dispositivo de aviso para cuando el almacenamiento de agua esté próximo a vaciarse.

- Debe incluir un puerto de carga que pueda conectarse y desconectarse.

- Debe ser impermeable a posibles líquidos que caigan sobre la mesa (bebidas, limpia polvo, etc).

- El precio máximo de fabricación debe ser de $500 €$.

- Debe incluir algún sensor de humedad que indique la humedad del ambiente de la habitación si se le solicita.

- Aspecto visual exterior agradable y moderno.

A partir de esta información, el trabajo será desarrollar de forma completa las siguientes tareas:

- Planificación de proyecto de diseño y desarrollo del producto. Definición de métodos de gestión, etapas y roles. Gestión del Equipo.

- Análisis del producto y ampliación de la definición de los requisitos funcionales junto con los del promotor, del usuario y del diseñador.

- Elaboración del diseño conceptual y del formal del producto. Definición del Alcance.

- Selección de materiales para los componentes del producto, considerando nuevos materiales.

- Desarrollo del metaplan de procesos de fabricación con selección razonada y justificada.

- Modelar los elementos básicos del producto y realizar análisis y simulación con las herramientas adecuadas. Analizar e interpretar los resultados.

- Diseño de detalle del producto incluyendo documentación gráfica (planos) con lista de materiales, conjunto y detalle de piezas principales.

- Documentar el Trabajo de forma legible.

\subsection{Entregas del proyecto}

Para potenciar la función de guía del profesorado durante el desarrollo del proyecto, se plantearon tres seminarios a lo largo del semestre, que implican una breve exposición del trabajo realizado hasta ese momento por parte de los grupos de trabajo. Tras cada seminario el profesorado valorará las entregas parciales, dando una corrección que sirva de orientación para futuras etapas.

En cada uno de los seminarios se valoraron diferentes aspectos del trabajo asignando unas rubricas a cada uno de los puntos. Las rúbricas se proporcionaban a los alumnos a principio de curso para que conocieran el proceso de evaluación. Un ejemplo de la rúbrica del primer seminario puede verse en la Tabla 1. Los aspectos a evaluarse se establecieron en base a las tareas a desarrollar en el proyecto hasta dicho seminario 
y las competencias marcadas en la asignatura. Las rúbricas han sido utilizadas en trabajos similares obteniendo buenos resultados (Toledo, 2018).

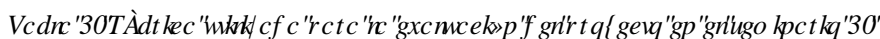

\begin{tabular}{|c|c|c|c|c|c|}
\hline ENTREGABLE 1 & 4 & 3 & 2 & 1 & $\mathbf{0}$ \\
\hline $\begin{array}{l}\text { 1. Recopilación de } \\
\text { información y estudio } \\
\text { de la solución/producto } \\
\text { a diseñar y desarrollar }\end{array}$ & $\begin{array}{l}\text { Se define el } \\
\text { problema y se } \\
\text { analizan los } \\
\text { productos del } \\
\text { mercado }\end{array}$ & $\begin{array}{l}\text { Se define el } \\
\text { problema y se } \\
\text { analizan algunos } \\
\text { productos del } \\
\text { mercado }\end{array}$ & $\begin{array}{l}\text { Se define } \\
\text { vagamente el } \\
\text { problema y se } \\
\text { analizan algunos } \\
\text { productos del } \\
\text { mercado sin } \\
\text { criterios }\end{array}$ & $\begin{array}{l}\text { NO se define el } \\
\text { problema y NO } \\
\text { se analizan } \\
\text { algunos } \\
\text { productos del } \\
\text { mercado sin } \\
\text { criterios }\end{array}$ & $\begin{array}{l}\text { No se } \\
\text { incluye }\end{array}$ \\
\hline $\begin{array}{l}\text { 2. Análisis de requisitos. } \\
\text { Estudio de modelos del } \\
\text { mercado según el tipo, y } \\
\text { comparación de } \\
\text { alternativas } \\
\text { conceptuales de diseño, } \\
\text { indicando ventajas e } \\
\text { inconvenientes }\end{array}$ & $\begin{array}{l}\text { Los requisitos se } \\
\text { analizan } \\
\text { cuidadosamente y de } \\
\text { forma confiable. Se } \\
\text { comparan las } \\
\text { alternativas }\end{array}$ & $\begin{array}{l}\text { Los requisitos se } \\
\text { analizan de forma } \\
\text { poco confiable. Se } \\
\text { comparan las } \\
\text { alternativas }\end{array}$ & $\begin{array}{l}\text { Los requisitos se } \\
\text { analizan de forma } \\
\text { poco confiable. } \\
\text { NO se comparan } \\
\text { las alternativas }\end{array}$ & $\begin{array}{l}\text { Los requisitos } \\
\text { NO se analizan. } \\
\text { NO se comparan } \\
\text { las alternativas }\end{array}$ & $\begin{array}{l}\text { No se } \\
\text { incluye }\end{array}$ \\
\hline $\begin{array}{l}\text { 3. Definición del Ciclo } \\
\text { de Vida. Etapas y fases } \\
\text { del producto, } \\
\text { descripción y factores }\end{array}$ & $\begin{array}{l}\text { Se realiza una } \\
\text { propuesta de Fases } \\
\text { del Ciclo de Vida y } \\
\text { las interrrelaciones. } \\
\text { Se describen posibles } \\
\text { factores de impacto } \\
\text { ambiental en cada } \\
\text { una }\end{array}$ & $\begin{array}{l}\text { Se realiza una } \\
\text { propuesta de Fases } \\
\text { del Ciclo de Vida } \\
\text { sin interrrelaciones. } \\
\text { Se describe algún } \\
\text { factor de impacto } \\
\text { ambiental }\end{array}$ & $\begin{array}{l}\text { Se realiza una } \\
\text { propuesta de Fases } \\
\text { del Ciclo de Vida } \\
\text { incoherente. Se } \\
\text { describe algún } \\
\text { factor de impacto } \\
\text { ambiental }\end{array}$ & $\begin{array}{l}\text { Se realiza una } \\
\text { propuesta de } \\
\text { Fases del Ciclo } \\
\text { de Vida } \\
\text { incoherente. NO } \\
\text { se describen } \\
\text { factores de } \\
\text { impacto } \\
\text { ambiental }\end{array}$ & $\begin{array}{l}\text { No se } \\
\text { incluye }\end{array}$ \\
\hline $\begin{array}{l}\text { 4. Diseño conceptual. } \\
\text { Bocetos propuestos }\end{array}$ & $\begin{array}{l}\text { Se realizan una serie } \\
\text { de Bocetos de } \\
\text { calidad artística }\end{array}$ & $\begin{array}{l}\text { Se realizan una serie } \\
\text { de Bocetos de poca } \\
\text { calidad artística }\end{array}$ & $\begin{array}{l}\text { Se realiza un } \\
\text { Boceto de poca } \\
\text { calidad artística }\end{array}$ & $\begin{array}{l}\text { NO se realizan } \\
\text { Bocetos } \\
\text { adecuados }\end{array}$ & $\begin{array}{l}\text { No se } \\
\text { incluye }\end{array}$ \\
\hline $\begin{array}{l}\text { 5. Selección de } \\
\text { Materiales y procesos }\end{array}$ & $\begin{array}{l}\text { Se analizan y } \\
\text { describen nuevos } \\
\text { materiales con } \\
\text { propiedades y } \\
\text { funciones buscando } \\
\text { aplicación a } \\
\text { componentes del } \\
\text { producto }\end{array}$ & $\begin{array}{l}\text { Se analizan y } \\
\text { describen nuevos } \\
\text { materiales con } \\
\text { propiedades y } \\
\text { funciones } \\
\text { genéricamente }\end{array}$ & $\begin{array}{l}\text { Se describen } \\
\text { nuevos materiales } \\
\text { genéricamente }\end{array}$ & $\begin{array}{l}\text { Se describen } \\
\text { materiales pero } \\
\text { no constituyen } \\
\text { ninguna } \\
\text { novedad } \\
\text { apreciable }\end{array}$ & $\begin{array}{l}\text { No se } \\
\text { incluye }\end{array}$ \\
\hline
\end{tabular}

\subsection{Cronograma de trabajo}

Durante la realización del proyecto, se esperaba que los estudiantes realizaran parte de su investigación fuera de las horas lectivas, mientras que el tiempo en clase se dedicaba a vigilar la evolución de cada grupo y a ayudarles a continuar con su trabajo. Para facilitar la situación de liderazgo del estudiante y evitar que la acumulación de trabajo en fechas próximas a las entregas, se estableció al principio del curso un cronograma de desarrollo de las tareas mencionadas en el punto 3.3. El cronograma de trabajo se puede ver en la Tabla 2. La conocimiento del plan de trabajo al principio de curso permite generar una mejor organización de las seisones de trabajo por parte de los alumnos como se ha observado en otros estudios similares (Ayala-cabrera, 2020) 


\section{Resultados}

A continuación, se muestran los resultados obtenidos en el desarrollo de esta innovación. Por una parte, se han desarrollado resultados directos de la actividad diseñada y, por otro lado, resultados obtenidos a partir de la innovación midiendo el impacto en el aula en los alumnos de la asignatura.

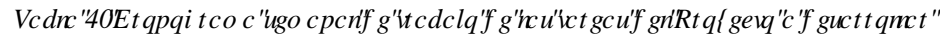

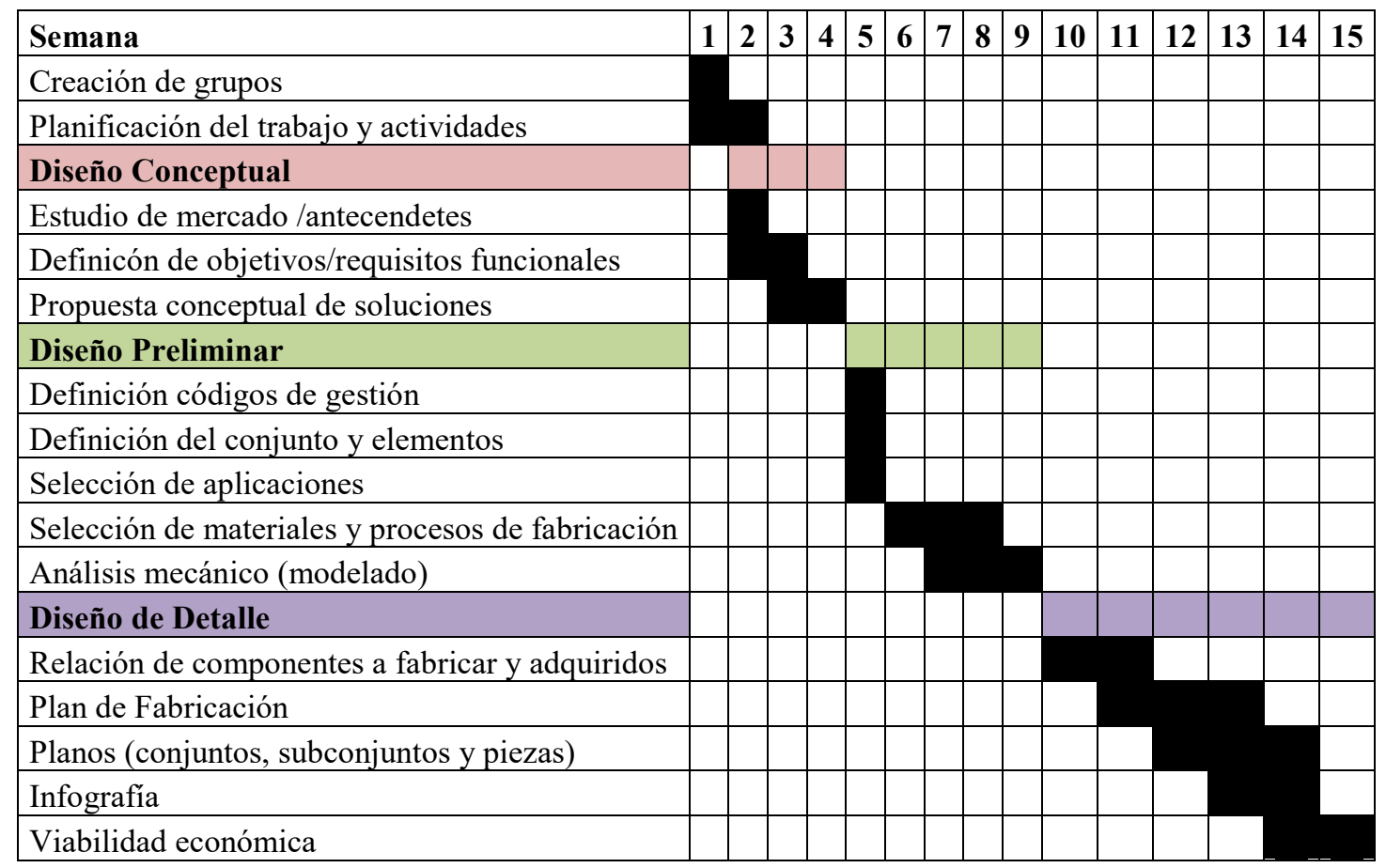

\subsection{Resultados del profesorado}

Para determinar las limitaciones y necesidades de los alumnos en la asignatura los profesores realizaron diversas reuniones de planificación de la actividad. Estas reuniones han sido uno de los puntos más importantes realizados durante el desarrollo de esta innovación ya que han permitido establecer las bases del entendimiento entre los profesores unificando y clarificando conceptos, además de establecer las bases para alcanzar las distintas competencias a considerar en ambas asignaturas.

Como resultados, se obtuvieron las limitaciones del proyecto presentado, el cronograma de trabajo, y la generación de rúbricas para evaluar la adquisición de las diversas competencias a alcanzar durante esta actividad.

\subsection{Resultados del alumnado}

El resultado de la actividad planteada a los estudiantes de máster en la asignatura de Ciclo de Vida del Producto fue el documento con el proyecto completo de la mesa humidificadora, purificadora y deshumidificadora de aire con filtro HEPA, que incluía desde la selección de materiales y procesos de fabricación, hasta los planos y montaje de la misma. Todos los grupos de trabajo completaron la experiencia con éxito, presentado trabajos de alto nivel, que fueron presentados al concurso - $D P H \mathbb{V}^{\prime} \mid \mathrm{VRQ} \$ \mathrm{ZDC}$.

El material que desarrollaron fue corregido y adaptado durante los seminarios fijados, de tal manera que sirviera de guía de mejora para los estudiantes. Se observaron tres resultados destacables. En primer lugar, 
se observó que aquellos estudiantes que estaban menos interesados en la materia se vieron motivados por los compañeros que tenían habilidades en campos diferentes al suyo. De esta manera, el carácter multidisciplinar el proyecto, permitió que los alumnos aprendieran de sus compañeros a la vez que destacaban en aquellos temas más relacionado con su área. En segundo lugar, el alumnado se muestró más receptivo en el aula ya que poder relacionar los conceptos teóricos con problemas reales les permitía asimilan mejor los conceptos teóricos. Finalmente, al situar al alumno en el centro del aprendizaje y tomar un papel más activo en el proceso de aprendizaje se mejoró la relación y la participación en la comunicación con el profesor.

Para tener unos resultados más objetivos se planteó a los alumnos un cuestionario sobre las siguientes preguntas:

- Aparte de la documentación de la propia asignatura facilitada por el profesorado, indicar el tema sobre el que más se ha buscado información complementaria en el ámbito de la asignatura.

- Indicar qué tipo de fuentes bibliográficas, fondos electrónicos o informes se han consultado durante la preparación de la asignatura en el curso en el ámbito del trabajo.

- Indicar las aplicaciones y herramientas utilizadas para esta asignatura y los cursos de autoformación realizados.

A la vista de las distintas respuestas obtenidas de los alumnos, se comprobó que el alumno profundizaba en temas donde su conocimiento estaba más limitado, siendo normalmente el contrario al de la carrera de procedencia, lo que permita desarrollar habilidades complementarias a las adquiridas durante el grado.

También se observó que los alumnos aprendían a trabajar con recursos electrónicos reales, ya sean páginas webs directas de fabricantes, catálogos de venta de herramientas o máquinas de mecanizado, o software específico de diseño o selección de materiales. En algunos casos, el alumno se puso en contacto incluso con los comerciales de la empresa para solicitar costes de las piezas o componentes necesarios para el proyecto, lo que supuso un contacto directo con la empresa.

Grupo sin ABP

MEDIAS

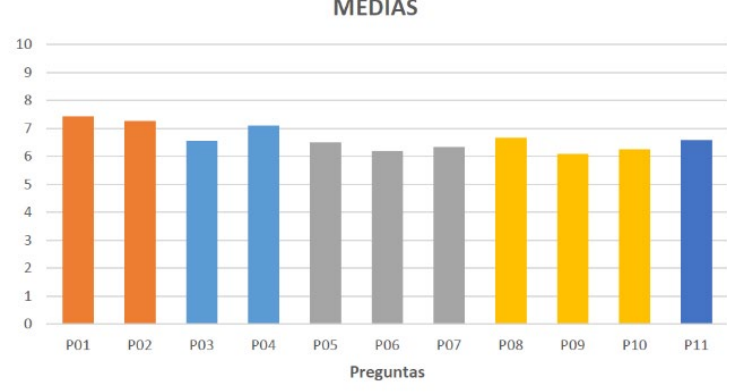

Grupo con $\mathrm{ABP}$

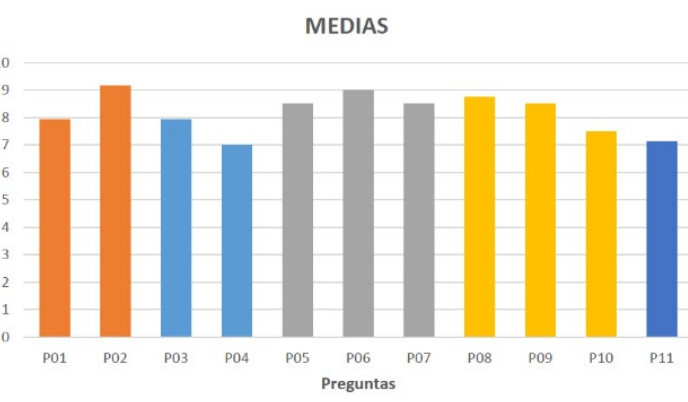

Preguntas

Conocimiento de la materia $\quad$ Organización y planificación

— Desarrollo/Metodología docente 1 Motivación/Interacción/Ayuda

- Satisfacción general

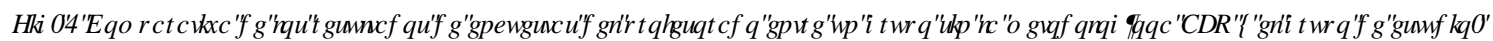


Finalmente, se pidió a los alumnos que evaluaran la satisfacción con el profesorado y la asignatura en global. En la Figura 2 se conbtrastan estos resultados frente a un grupo de estudiantes similar que cursa una asignatura muy similar en otro máster de la universidad, donde no se aplicó la metodología ABP y se mantuvo la metodología tradicional. Se puede observar en esta Figura como los alumnos quedaron bastante satisfechos con la metodología docente $(8.75 / 10)$, siendo muy distinta a la que están acostumbrados, consiguiendo también una interacción alta con el profesorado que les ha servido de ayuda durante la realización del proyecto $(8.2 / 10)$.

\section{Conclusiones}

El planteamiento de la asignatura "Ciclo de vida del producto" y las competencias a evaluar de la misma, ha hecho que la experiencia previa de los profesores recondujera la asignatura a una orientación más aplicada. En este sentido, la metodología de Aprendizaje Basado en Proyectos permite al mismo tiempo desarrollar y evaluar tanto las competencias específicas de la asignatura como desarrollar las competencias

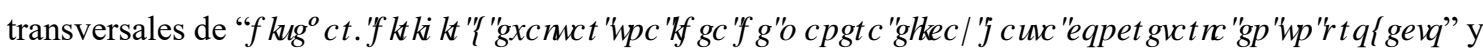

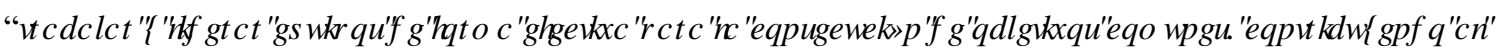
GHDURCASHURQDO ISLRIHMRQDOGHIRVP LMP RV”.

Las impresiones por parte del alumnado son muy positivas, acogiendo de forma activa este tipo de metodologías, consiguiendo que los alumnos alcancen resultados de aprendizaje establecidos con un nivel mayor que el que obtienen con las clases magistrales y resolución de ejercicios individuales. Debido a que se propone trabajar con alumnos de distintos grados y con distinto grado de madurez académica, se ha proporcionado más libertad a los estudiantes para profundizar en la realización del proyecto distribuyendo las tareas en función de sus intereses como se ha visto en estudios similares en la literatura (Naranjo, 2019).

Las evaluaciones de los estudiantes mediante las rúbricas parecen indicar que mediante esta metodología logran un mayor desarrollo de habilidades profesionales (Toledo, 2018). Especialmente los relacionados con el análisis de situaciones profesionales, indagación, resolución de problemas, toma de decisiones, autonomía para aprender, trabajo en equipo y comunicación. Es especialmente interesante poner de relieve el hecho de que la capacidad de resolución de problemas también se desarrollaría más mediante el Aprendizaje Basado en Proyectos que con el aprendizaje basado únicamente en problemas (Abella, 2020). De acuerdo con los resultados de nuestro estudio, el Aprendizaje Basado en Proyectos debe considerarse como una opción metodológica preferencial para el desarrollo de competencias profesionales en la educación en ingeniería.

\section{Referencias}

ABELLA, V., AUSÍN, V., DELGADO, V., CASADO, R. (2020). "Aprendizaje Basado en Proyectos y Estrategias de Evaluación Formativas: Percepción de los Estudiantes Universitarios". En: 5HLWD, EHRDP HUFDQDGH( YDQDFlyQ ( GXFDUMD, Vol. 13 (1), 93-110.

$<$ https://doi.org/10.15366/riee2020.13.1.004>

AYALA-CABRERA, J., PÉREZ-RÀFOLS, C., NUÑEZ, O., SERRANO, N. (2020). "Implementación del Aprendizaje Basado en Proyectos en laboratorios de química analítica del Grado de Química". En: \$ YDQFHHQL\&IHQFDVH, QJ HQIHED, Vol. 11 (2), 31-40.

< http://diposit.ub.edu/dspace/bitstream/2445/166962/1/699732.pdf>

BLUMENFELD, P.C. et al (1991). "Motivating project-based learning: Sustaining the doing, supporting the learning", en ( GXFDURQDOB W FKRCDJLW, 26 (3 \& 4), 369-398.

$<$ http://formacion.intef.es/pluginfile.php/37233/mod_resource/content/1/PDF/5_AbP_bloq3_u1.pdf >

(c)) BY-NC-ND 2021, Universitat Politècnica de València

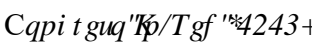




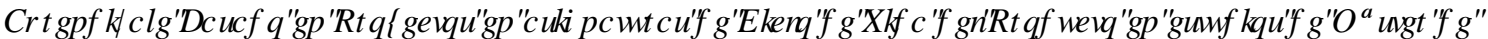 ' LMKR】 D) DEUFDFlyQ}

CUIÑAS I., MARIÑO-ESPIÑEIRA, P., FERNANDEZ-IGLESIAS, M., CAEIRO, M., COSTAMONTENEGRO, E., DIAZ-OTERO, F. (2016). "Evaluación de competencias con metodologías de aprendizaje basado en proyectos". En: \&RQJUHR, 15( ' प्र०पVValencia: Universitat Politècnica de València. 1-14.

$<$ https://web.ua.es/es/ice/jornadas-redes-2012/documentos/posters/245822.pdf >

ESTEPA, R.M. y ESTEPA, A. J. (2011). “Trabajar con la incertidumbre del mundo laboral: análisis de una experiencia

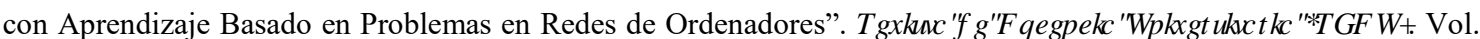
$9(2)$, pp. $213-232$.

$<\mathrm{http}: / /$ redaberta.usc.es/redu $>$

GARCIA, J., PEREZ, J. (2018). “Aprendizaje basado en proyectos: método para el diseño de Actividades". 5HLML 7HFQRQRJ TDI\&IHQFIDH, QQRIDFIY Q Vol. 10, pp. 37-63.

$<$ https://www.tecnologia-ciencia-educacion.com/index.php/TCE/article/view/194 >

GARRIGÓS, J. y VALERO-GARCÍA, M. (2012). "Hablando sobre Aprendizaje Basado en Proyectos con Júlia". 5HIWDGH' RFHQFD8 QYYHWWUDD5( ' 8 [, Vol. 10 (3), 125-151.

$<$ http://red-u.net/redu/index.php/REDU/article/view/484>

GÓMEZ-PABLOS V.B., DEL POZO M. M. y MUÑOZ-REPISO A.G.V. (2016) "Aprendizaje Basado en Proyectos (ABPr) incorporando las TIC: ventajas e inconvenientes desde la experiencia del profesorado en ejercicio", 7HFQRQJ tDD

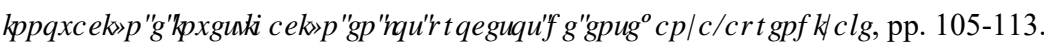

$<\mathrm{https} / /$ dialnet.unirioja.es/servlet/articulo?codigo $=6015791>$

GONÇALVES, S.R. (2014). "Preparing Graduates for Professional Practice: Findings from a Case Study of Projectbased Learning (PBL)”. 3 LRFHADIL6RFDODQCH\%HDVIRLDO6FHQFH, Vol. 139 (22), pp. 219-226.

$<$ https://doi.org/10.1016/j.sbspro.2014.08.064>

MANCHADO, E. y BERGES, L. (2013). "Una experiencia de PBL en Grado de Ingeniería de Diseño Industrial,

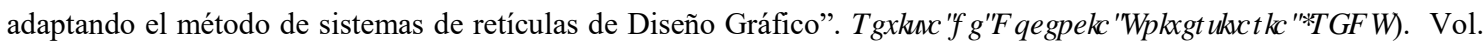
11, pp. 19-46.

$<$ http://red-u.net/redu/index.php/REDU/article/view/564/pdf $>$

METTAS, A.C., CONSTANTINOU, C.C. (2007). "La academia en acción: aprendizaje basado en proyectos en entornos universitarios' en ,QUAQDWRQDO RXLQDORID7HKQRQRJ IDOQJ' HMJQ( GXFDURQ Vol. 18, pp. 79-100. $<$ https://doi.org/10.1007/s10798-006-9011-3 >

NARANJO, A., CORREA, F. (2019). "The technology fair: a project-based learning approach for enhancing problem solving skills and interest in design and technology education' 55 HLWW\% ROMQ5 HaSH Vol. 9 (1), pp. 70-78.

$<$ https://revista.redipe.org/index.php/1/article/view/893>

PEREIRA M.A. (2015). TI HOP HQRVV HMQFIDOND GHD \$\%3. https://cedec.intef.es/7-elementos-esenciales-del-abp/ [Consulta: 16 de marzo de 2021]

TOLEDO, P., SÁNCHEZ, J.M. (2018). "Aprendizaje basado en proyectos: una experiencia universitaria' en

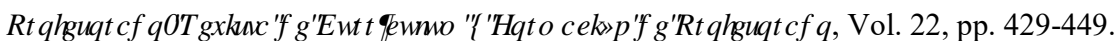

$<$ https://recyt.fecyt.es/index.php/profesorado/article/view/66383 >

ZAFRA, C.A. (2020). Aprendizaje de la automatización industrial en tiempos de pandemia. Una Experiencia virtual de aprendizaje basado en proyectos. Trabajo Final de Grado. Bogotá: Universitaria Agustiniana,

$<$ https://repositorio.uniagustiniana.edu.co/handle/123456789/1314> [Consulta: 19 de mayo 2020] 